\title{
Automated Detection of Diabetic Retinopathy using Deep Residual Learning
}

\author{
Md Ashikur Rahman \\ Senior Software Engineer, \\ Smart Technologies BD Ltd. \\ Smart Jahir Tower, H \# 217, Begum \\ RokeyaSarani, Dhaka \\ 1217, Bangladesh
}

\author{
Md Arifur Rahman \\ QA Automation Engineer (Remote) \\ Crossover \\ Austin, Texas, USA
}

\author{
Juena Ahmed Noshin \\ Assistant Professor, American \\ International University-Bangladesh \\ (AIUB) \\ 408/1, Kuratoli, Khilkhet, Dhaka \\ 1229, Bangladesh
}

\begin{abstract}
Significant amount of people suffer from Diabetic Retinopathy (DR), which is one of the major causes of vision loss. The incidence of this disease is even higher due to not being diagnosed at the right time. On numerous occasions, due to neglect and poor care, diabetic retinopathy can lead to significant damage to the eyes. That is why, early diagnosis of eye diseases, proper treatment and care for the disease can prevent vision loss. Referral of eyes with diabetic retinopathy for advanced assessment and treatment would aid in reducing the chances of vision loss, allowing proper diagnoses. The purpose of this study is to develop resilient and flexible diagnostic techniques for the detection of DR and to identify dynamic DR grading using residual networks to facilitate the network training that are significantly intense than previously used networks. Even though lots of research has been done on DR, its identifications remains challenging due to time and space complexity along with higher accuracy specificity. Here, a residual learning framework has been proposed that overcomes the challenges while efficiently detecting DR. Hence, using a high-end Graphics Processor Unit (GPU) the model has been trained on the publicly available Kaggle dataset and empirical evidence has been provided in order to support the results with a sensitivity of $95.6 \%$ and an accuracy of $93.20 \%$.
\end{abstract}

\section{General Terms}

Machine Learning, Convolutional Neural Network

\section{Keywords}

Diabetic Retinopathy, Deep Neural Network, Residual Learning, Image Recognition

\section{INTRODUCTION}

Different methods of detecting abnormalities in Diabetic Retinopathy (DR) have been studied in various research works using deep learning which also lead to many solutions being provided. The methods suggested in these research activities can assist ophthalmologists in eliminating vascular and optic discs. In [5], an approach for the Automatic Detection of Diabetic Retinopathy (ADDR) in color fundus images has been discussed. This technique utilizes preprocessing, feature extraction, and integration of classification algorithms. The effectiveness of the automated system has been evaluated on the basis of sensitivity and specificity in this approach. On the other hand, an automated diagnosis system has been proposed for detecting DR from 250 selected images in [7]. Here, Match Filter Response (MFR) has been used to reduce noise in images. Afterwards, Fuzzy C-Mean Clustering has been applied to these images to segment the blood vessels. After preprocessing, techniques like Probabilistic Neural Network, Bayes Theory and Support Vector Machine have been used to compare their performance.

Deep Residual Learning (DRL) has a spectacular record in the area of image analysis, including medical imaging. Over the past few years, updated architectures on the Residual Networks i.e. ResNet 34, ResNet 50, ResNet 152 etc. have been introduced and studied in several researches. This work employs a residual learning framework called ResNet 50, a 50-level residual network used to train and facilitate networks that are significantly deeper than previously used networks. However, here explicit learning-like layers has been formulated with reference to level inputs rather than learning abstract functionality. This research shows that this pretrained model is very useful in determining DR considering the large number of parameters in the dataset and the time complexity. On top of that, a high-end graphics processor unit (GPU) has been used to train the model in the dataset which eventually speeds up the process. In the end, comprehensive and extensive empirical evidence has been provided showing that in detection of DR the proposed residual learning framework more specifically ResNet 50 can achieve higher precision from sufficiently increased depths with lower time and space complexity.

\section{RELATED WORK}

A lot of researchers have conducted studies regarding detection of Diabetic Retinopathy (DR) using various algorithms, methodologies, techniques and procedures. Amongst them, some relevant works have been discussed here which have direct correlation with our proposed approach.

In [1] an automated approach to the identification of the presence of diabetic retinopathy from color fundus images of the retina has been proposed. This research incorporated a three layered artificial neural network (ANN) and support vector machines (SVM) to classify the retinal images. The proposed method can produce a classification accuracy of 97.2\% using ANN and $98.1 \%$ using SVM.

In [2] researchers have used the green channel of the fundus RGB image for obtaining traces of blood vessels. The developed algorithm utilized morphological operation to smoothen the background ensuring that the veins have better visibility. Contrast enhancement, background exclusion and thresh holding have been used in this approach. The proposed techniques have been applied on some publicly available databases and images from eye hospitals. Experimental results obtained from implementing the proposed algorithm has 
shown it to be a highly effective method for classifying retinal blood vessels. Moreover, the approach is deemed best suited for applications that require faster processing.

The abnormalities occurring for Diabetic Retinopathy have been studied in [3]. The methodology propose here can help eye specialists to eliminate blood vessels and optic disc. Another application of this proposed approach is that it can detect MAs and Exudates in Diabetic Retinopathy screening process. All of the results obtained from implementation are evaluated in coordination with images obtained from specialists. An average accuracy of $99.6 \%$ has been achieved by the proposed method in detecting exudates from the retinal fundus images. The results from the experiments show that, the proposed approach is well suited for the early diagnosis of the Diabetic Retinopathy disease. Through thorough evaluation of the exudates and fovea region, the severity of DR can be identified and as a result vision loss in diabetic patients may be prevented.

Visual information of images has been enhanced using image processing techniques in [4] to increase the visibility of crucial anatomical structure. Machine learning methods have been applied on the dataset after the feature extraction phase to grade retinal images according to quality scale.

In [5], a method for the automatic detection of Diabetic Retinopathy (ADDR) in color fundus images has been discussed. In this technique a concoction of preprocessing, feature extraction and classification algorithms have been used. In this approach performance of the automated system has been assessed based on Sensitivity and Specificity which is $94.44 \%$ and $87.5 \%$, respectively.

In [6], the researchers aimed to determine the sensitivity and specificity of the Iowa Detection Program (IDP) in detecting referable diabetic retinopathy (RDR). The results from this research indicate that automated detection has become ready for clinical use.

An automated diagnosis system has been proposed in [7] for diabetic retinopathy on 250 selected images. Matched filter response (MFR) has been used to reduce the noise in the images. To segment the blood vessels in these images Fuzzy c-means clustering has been applied. After preprocessing phase, techniques such as PNN, Bayes Theory and SVM are used to compare their performance. In the final phase, images are classified according to grade. The accuracy rate of PNN and SVM are $89.6 \%$ and $97.6 \%$ respectively.

The research in [8] has aimed focus on screening for presence or absence of Diabetic Retinopathy (DR). From analysis phase of this work it has been found that outreach DR screening is an effective alternative to screening that uses medical specialist photographer. The outreach model seems to show enormous potential to become an economic way of preventing blindness among diabetic patients.

The researchers in [9] used a random forest methodology to perform classification analysis of DR fundus photography data. The data analysis clearly indicates that the proposed work outperforms logistic regression in almost all situations provided during the experiments.

In [10] researchers observed that Telemedicine screening by the primary care physician may lead to a better approach in managing DM as it can improve screening process and may ultimately prevent vision threatening DR.

The paper in [11] proposed a novel method for the automated identification of exudates pathologies in retinopathy fundus images based on computational intelligence technique. The proposed approach uses a unique sequential execution of morphological operators to extract fundus image features. Afterwards, the selected features are passed into the wellknown support vector machine (SVM) classifier which classifies the images into normal and abnormal classes. Real time and publicly available database analysis has shown very encouraging performance metrics of the proposed methodology in terms of sensitivity, specificity and accuracy.

In [12], an automatic image processing algorithm for detection of (Hard Exudates) HEs has been proposed based on retinal image analysis. The proposed algorithm consists of image preprocessing, enhancement, feature extraction, classification and post-processing. However, a limitation occurred in this research as the performance of automatic HE detection algorithms on retinal images could not be determined due to unavailability of public database.

An automated screening system has been developed in [13] to analyze digital color retinal image. Pre-processing of color image, identification of main retinal components and recognition of diabetic retinopathy are the consequent steps of this approach. To identify retinal lesions the researchers had defined hard exudates, hemorrhage and micro aneurysms. Detection rate of hard exudates, hemorrhage and micro aneurysms for sensitivity and specification are $88.5 \%$ and $99.5 \%, 77.5 \%$ and $88.7 \%, 77.5 \%$ and $88.7 \%$ respectively.

This project [14] has been developed for Diabetic Retinopathy screening based on examination of tear fluid biomarker changes. The machine learning algorithms Support Vector Machine (SVM), Recursive partitioning, Random Forest, Naïve Bayes, Logistic regression, K-Nearest Neighbor have been applied here. The performance of this project reached a markup of $74 \%$ sensitivity and $48 \%$ specificity.

The results obtained from the paper [15] demonstrates that deep neural networks can be trained using large data sets to identify diabetic retinopathy or diabetic macular edema in retinal fundus images with high sensitivity and high specificity. The proposed automated system offers several advantages such as consistency of interpretation, high sensitivity, high specificity and near instantaneous result generation. In this study the sensitivity reached a score of $97.5 \%$.

The research work in [16] proposed a fully data focused artificial intelligence based grading algorithm to screen fundus photographs obtained from diabetic patients and also identify cases that should be referred to an eye specialist for further evaluation and treatment. The implementation of such an algorithm can positively impact diabetic patients on global basis i.e. it could drastically reduce the rate of vision loss attributed to DR worldwide. The proposed model achieved a whopping $94 \%$ sensitivity and $98 \%$ specificity respectively.

In [17], using deep learning the researchers demonstrated the extraction and quantification of multiple cardiovascular risk factors from retinal images. The proposed methodology has achieved an AUC performance of 0.7 .

The researchers in [18] developed a deep-learning algorithm for automated detection of Diabetic Retinopathy (DR). The proposed model showed sensitivity and specificity of $96.8 \%$ and $87.0 \%$ respectively.

A Deep Learning System (DLS) for screening Diabetic Retinopathy and related eye diseases has been developed in [19]. The proposed methodology identified that the referable 
diabetic retinopathy AUC: 0.93, vision-threatening AUC: 0.958 and glaucoma AUC: 0.942 respectively.

A deep Convolutional Neural Network-based algorithm has been proposed in [20] for image classification. Data Augmentation has been used in this research for translation, stretching, rotation and flipping the image data set. The use of convolutional neural network-based image classification can significantly reduce the computation time which combined with data augmentation approach contributed an accuracy rate of $94.5 \%$.

Researchers in [21] have introduced a deep learning based CNN method for classifying DR in fundus image. The final trained network had a sensitivity of $30 \%$, accuracy of $75 \%$ and specificity of $95 \%$. Moreover the algorithm has been deemed extremely efficient as it could classify thousands of images per minute.

A two staged Deep Convolutional Neural Network (DCNN) algorithm has been introduced in [22] to find the location and type of lesions in the fundus image. Following this proposed methodology yields an AUC value of 0.9687 .

This paper [23] proposed an approach to localize areas of images for lesions detection. Class Activation Maps (CAMs) has been modified for this research to make the architecture capable of weakly supervised localization. From experimental analysis the following results had been obtained i.e. $93.6 \%$ sensitivity and $97.6 \%$ specificity for this proposed approach.

The researchers of [24] aimed to find an approach to detect lesions or other biomarkers of DR using deep learning algorithm. In this approach lesion detector had been trained to supervise the image level labels.

In [25], a deep learning algorithm has been proposed using CNN in multi-categorical disease for fundus image analysis. This study has achieved better screening classification models to detect retinal abnormality with AUC of 0.903 , sensitivity of $80.3 \%$, and specificity of $85.5 \%$.

The researchers have developed an artificially intelligent algorithm using transfer learning techniques in [26]. This research achieved a sensitivity of $97.8 \%$, a specificity of $97.4 \%$ and a weighted error of $6.6 \%$

In this work [27], a novel framework Zoom-in-Net has been proposed which had achieved satisfactory performance on two datasets. Zoom-in-Net can generate attention maps which highlight the suspicious regions trained with image level supervisions only.

CNN architectures have been implemented in this paper [28] to detect diabetic retinopathy (DR). The novelty of this work is the discovery that diabetic retinopathy (DR) can be detected at the preprocessing stage.

In this paper [29], a methodology has been described for Diabetic Retinopathy detection from eye fundus images using a bag-of-visual-words (BoVW) method. The BoVW has been formulated as two neural networks that can be trained jointly. Area Under the Curve (AUC) has been determined as .97 for this model on the DR2 dataset.

Several researchers have conducted studies regarding noisy image detection. Among them some of the most recent advancement has been discussed here.

The research paper [30] describes a new AprilTag detection algorithm which improves upon the previous versions of the detector. The new version works in reducing the rate of false positives, increasing the detection rate, and reducing the amount of computing time needed for detection. This approach has made robust tag detection viable on computation limited systems.

It has been shown in [31] that adversarial samples crafted to fool DNNs can be effectively detected with two new features i.e. kernel density estimates and Bayesian neural network uncertainty estimates. These two features can handle complementary situations which can be combined as an effective defense mechanism against adversarial samples.

The main contribution of [32] is the consideration of the extraction of fine gray-variation information and the edge missing problem in the edge detection. The experimental results show that the proposed detector outperforms existing detectors in terms of the aggregate ROC curves and Pratt's FOM evaluations.

In [33], an end-to-end saliency learning framework has been proposed without the need of human annotated saliency maps in network training. Extensive experimental results on various benchmarking datasets prove the superiority of the proposed method.

An interesting approach has been proposed in [34] to detect drivable road area in monocular images. The proposed approach contributes in reducing human labeling effort which in turn makes training scalable.

The researchers in [35] propose a theoretically based study which aims to explain prevailing edge detection techniques. From the research work it has been deduced that canny edge detection performs better than all other detection techniques.

In this study [36], images corrupted by three types of noise such as gaussian, salt and pepper and speckle had been used. It is observed from the experimental analysis that for most of the noisy images, SIFT presents its stability but it is not very swift. SURF is the fastest one and its performance is close to SIFT. On the other hand HOG is advantageous in detecting edge and texture information of image.

Though in the recent years many research works have been done regarding detection of DR some problems still remain unsolved in this field. Our research focuses on solving the existing drawbacks in this domain by following a novel approach through which performance factors may reach a greater level of improvement.

\section{PROPOSED METHOD}

This section describes the proposed approach towards the detection of Diabetic Retinopathy (DR) using the residual learning framework. Initially, the structure of ResNet 50 where the dataset is trained and evaluated to build the proposed predictive model has been presented. Subsequently, as part of the data cleaning process, the uninformative data from the images in the training dataset has been removed. In short, the noise data has been reduced for greater sensitivity. Finally, in the result section the proposed model is evaluated with the test dataset which contains more than 35000 images and the results is discussed with extensive empirical evidence.

\subsection{Architecture}

Figure 1 represents the ResNet 50[23] model which consists of 5 stages each with a convolution and an identity block. Each convolution block has 3 convolution layers and each identity block also has 3 convolution layers. The ResNet 50 is capable of learning complex functions and presentations of inputs. Moreover, the architecture is capable of handling more 
than 25 million parameters which leads to better performance. However, a more inappropriate way of adding layers can ultimately have a negative impact on the final workplace. To overcome this challenge, a remedy had been proposed to this deterioration problem where the intermediate layers of a block could learn the referential function with the block input. In the event that no correction is required, the adjacent layers gradually learn to calibrate their weights toward zero so that the residual block represents an identity function.

\subsection{Noisy Image Reduction}

Images come with many elimination conditions such as blurred vision, poor or deficient color vision, floating and even partial loss of vision that can disrupt the results. However, to overcome this challenge the proposed method came up with a solution to detect and work on such noise data for greater sensitivity and accuracy. This is because as part of the process, the images in the training dataset have been trained and validated in order to detect inaccurate predictions so that specifically work on these images can be done to remove noisy portions. Lastly, these images have been converted to gray scales to reduce the light condition effects, unnecessary areas of the images have been cropped, and the images have been made to look better. This method reduces noise data by about $91 \%$ in the performed experiments. Figure 2 shows original noisy images in dataset which disrupts the accuracy of the results and on the other hand, after removing noisy data Figure 3 shows images after applying Ben Graham's preprocessing method in dataset which increases the accuracy of the results.

\subsection{Training}

The architecture shown in Figure 1 has been designed after studying the existing models mentioned in the literature review. For this Convolutional Neural Network(CNN) model, in order to train the experimental dataset, Kaggle's (https://www.kaggle.com/) dataset has been used which Kaggle provided with a large set of retina images taken using fundus photography under a variety of imaging conditions for a competition on Diabetes Retinopathy (DR). The training dataset contains more than 100000 images including noisy data of DR. A Pie Chart in Figure 4 represents several types of severity of diabetic retinopathy in training dataset. However, to implement the ResNet 50 shown in Figure 1, Keras with the TensorFlow back-end engine has been used, which provides a model level library, advancing high level building blocks for developing deep learning models, and for computing numbers, the model from CUDA by NVIDA have been used which provides a highly parallel architecture for graphics processors. Each 2-layer block in the model replaces the 3-level bottle block in a 34-layer net, resulting in 50-level ResNet 50. However, for each image in the training dataset, a $224 \times 224$ informative image was processed in our training dataset throughout the model. About $66.66 \%$ of the training dataset was used to train the model, and the rest of the dataset was used to validate the model. The learning rate starts at 0.11 and is divided by 22 while error plains and models are trained up to 21000 repetitions. A weight loss of 0.00011 and a speed of 0.96 had been used.

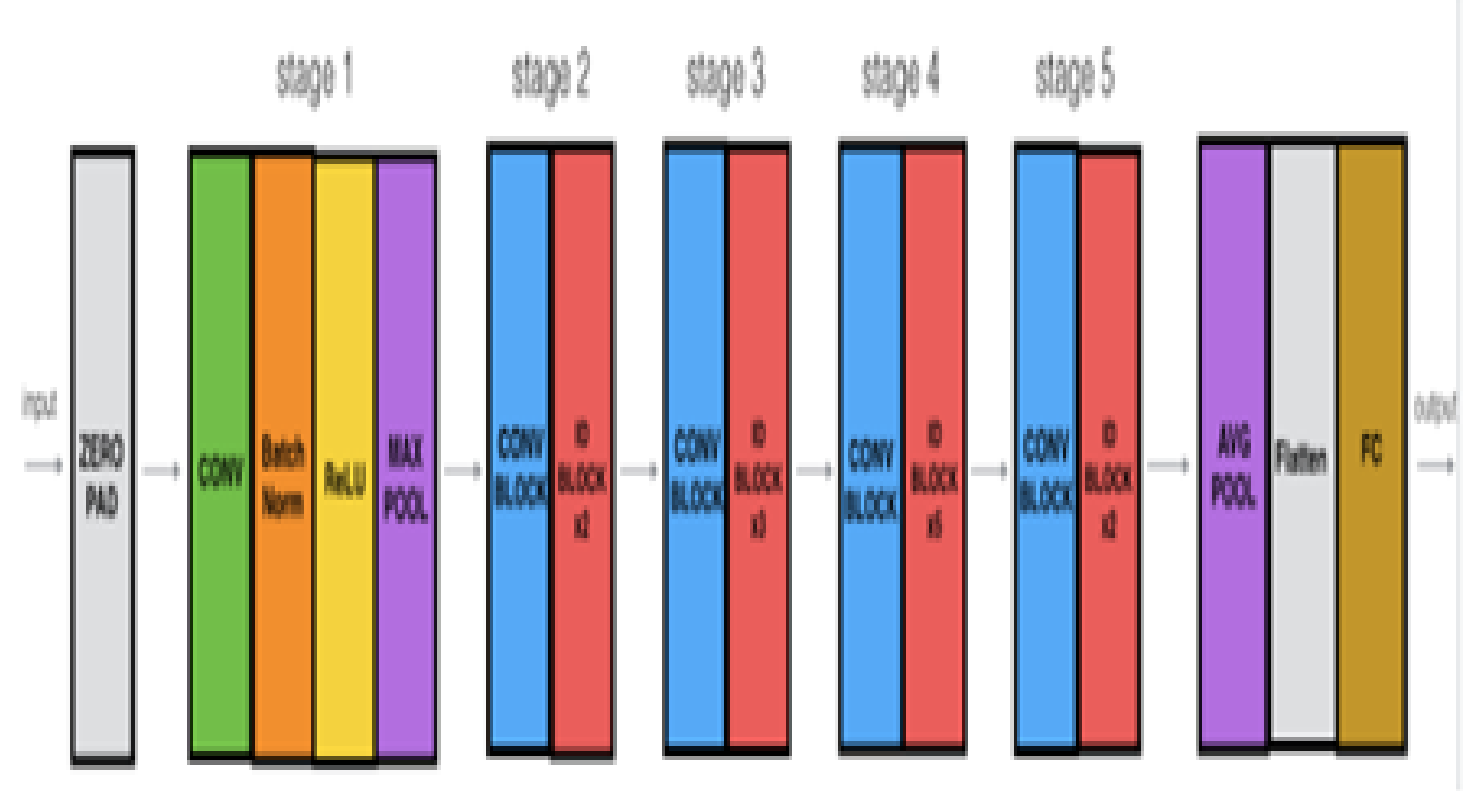

Figure 1: Compared to Plane Network, the above diagram represents Residual Network which is ResNet 50 Model with the architecture 


\section{$(-4.5,49.5,69.5,-7.5)$}
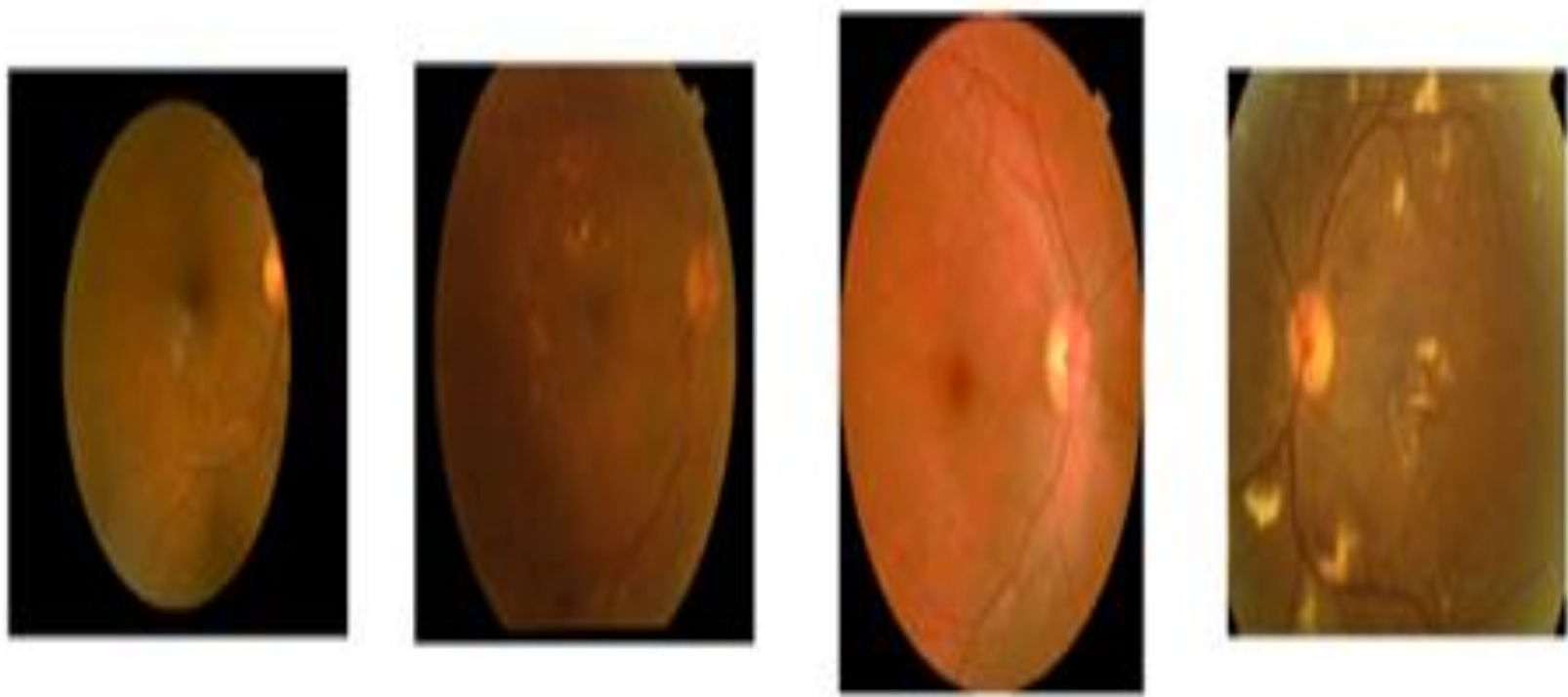

Figure 2: Shows original noisy images in dataset which disrupts the accuracy of the results

\section{$(-1.5,4.4 .5,49.5,+1.5)$}
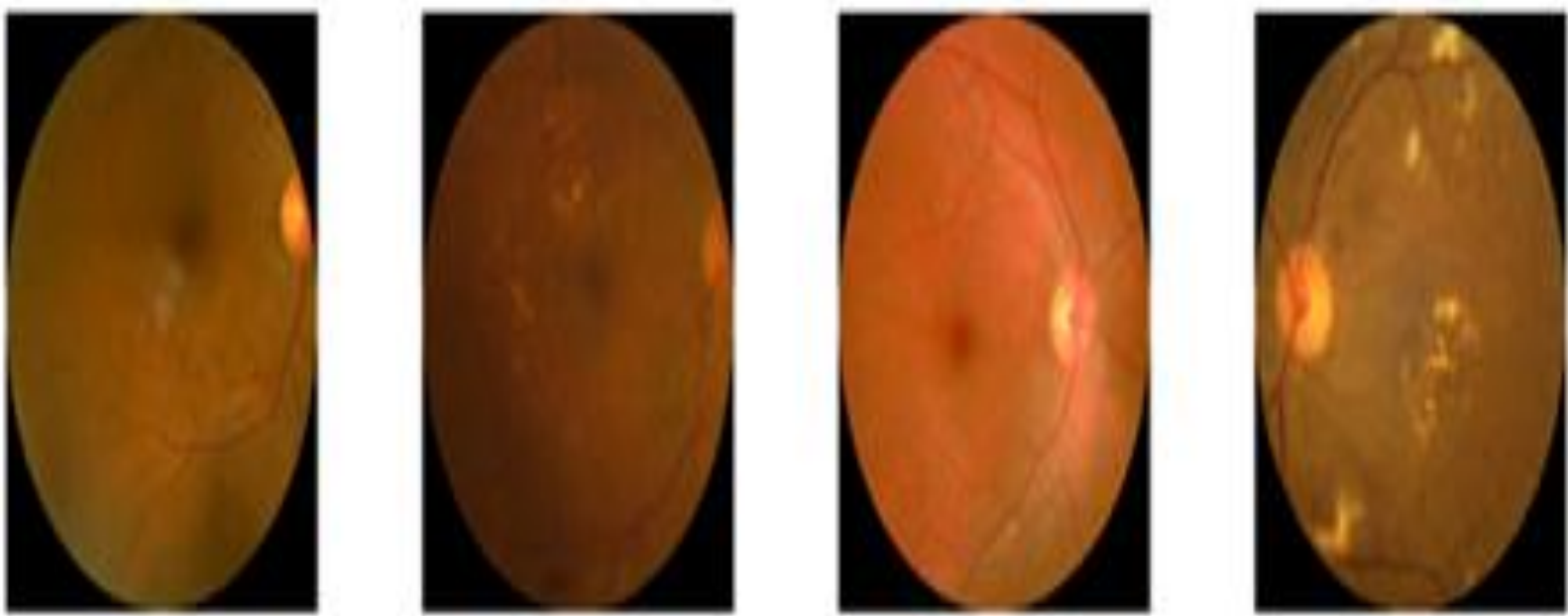

Figure 3: Shows images after applying Ben Graham's preprocessing method in dataset which increases the accuracy of the results 


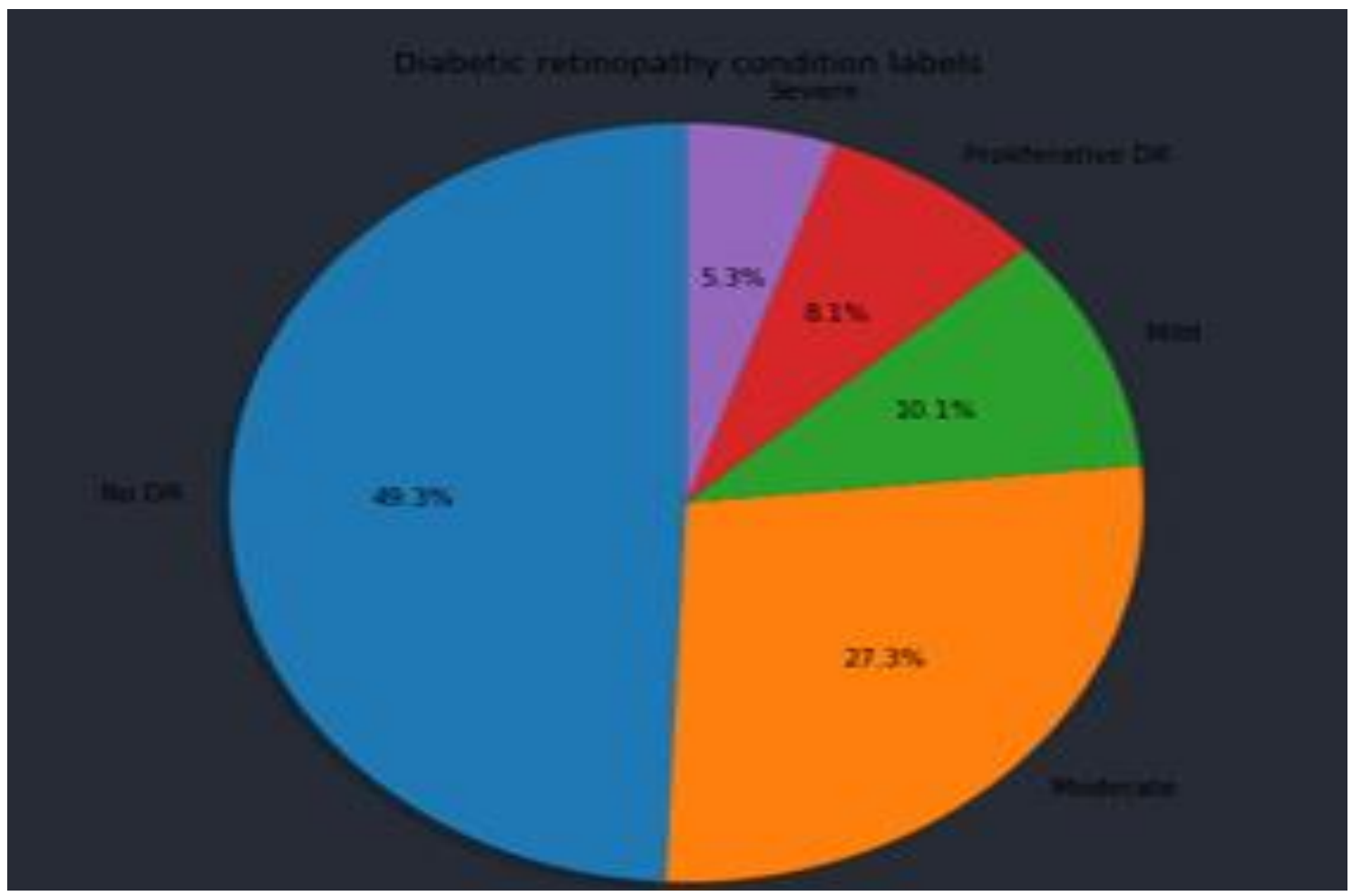

Figure 4: Pie Chart represents several types of severity of diabetic retinopathy in training dataset

\begin{tabular}{|c|c|c|c|c|c|}
\hline epoch & train_loss & valid_loss & accuracy & kappa_score & time \\
\hline o & 0.176382 & 0.196530 & 0.816940 & 0.872901 & $08: 53$ \\
\hline 1 & 0.157765 & 0.187075 & 0.830601 & 0.881923 & 08:59 \\
\hline 2 & 0.135613 & 0.224320 & 0.822404 & 0.910072 & $09: 01$ \\
\hline 3 & 0.130501 & 0.209721 & 0.836066 & 0.884820 & $08: 19$ \\
\hline 4 & 0.118669 & 0.215767 & 0.836066 & 0.880758 & $08: 10$ \\
\hline 5 & 0.101555 & 0.215721 & 0.819672 & 0.878971 & 08:06 \\
\hline 6 & 0.089405 & 0.225789 & 0.838798 & 0.8941 .46 & $08: 01$ \\
\hline 7 & 0.086767 & 0.2263 .44 & 0.814208 & 0.874250 & $08: 02$ \\
\hline 8 & 0.083010 & 0.196555 & 0.833333 & 0.884121 & $08: 05$ \\
\hline 9 & 0.066340 & 0.229426 & 0.836066 & 0.883139 & 08:09 \\
\hline 10 & 0.073857 & 0.237308 & 0.803279 & 0.881915 & $08: 01$ \\
\hline 11 & 0.062420 & 0.219833 & 0.836066 & 0.890136 & $08: 02$ \\
\hline 12 & 0.053 .462 & 0.240318 & 0.836066 & 0.894269 & $08: 29$ \\
\hline 13 & 0.050168 & 0.247789 & 0.855191 & 0.928774 & $09: 48$ \\
\hline 14 & 0.037567 & 0.249459 & 0.855191 & 0.916074 & $09: 52$ \\
\hline 15 & 0.036936 & 0.250609 & 0.833333 & 0.896963 & $09: 53$ \\
\hline 16 & 0.038270 & 0.248929 & 0.838798 & 0.909158 & $09: 57$ \\
\hline 17 & 0.0339 .49 & 0.268926 & 0.830601 & 0.898626 & $09: 0.4$ \\
\hline 18 & 0.029150 & 0.270 .460 & 0.841530 & 0.902966 & $08: 20$ \\
\hline 19 & 0.027710 & 0.256621 & 0.844262 & 0.901825 & $08: 14$ \\
\hline 20 & 0.030885 & 0.258093 & 0.844262 & 0.917061 & 08:09 \\
\hline 21 & 0.025538 & 0.255527 & 0.841530 & 0.913647 & 08:09 \\
\hline
\end{tabular}

Figure 5: Shows the values of training loss, validation loss along with quadratic kappa, considering the time 


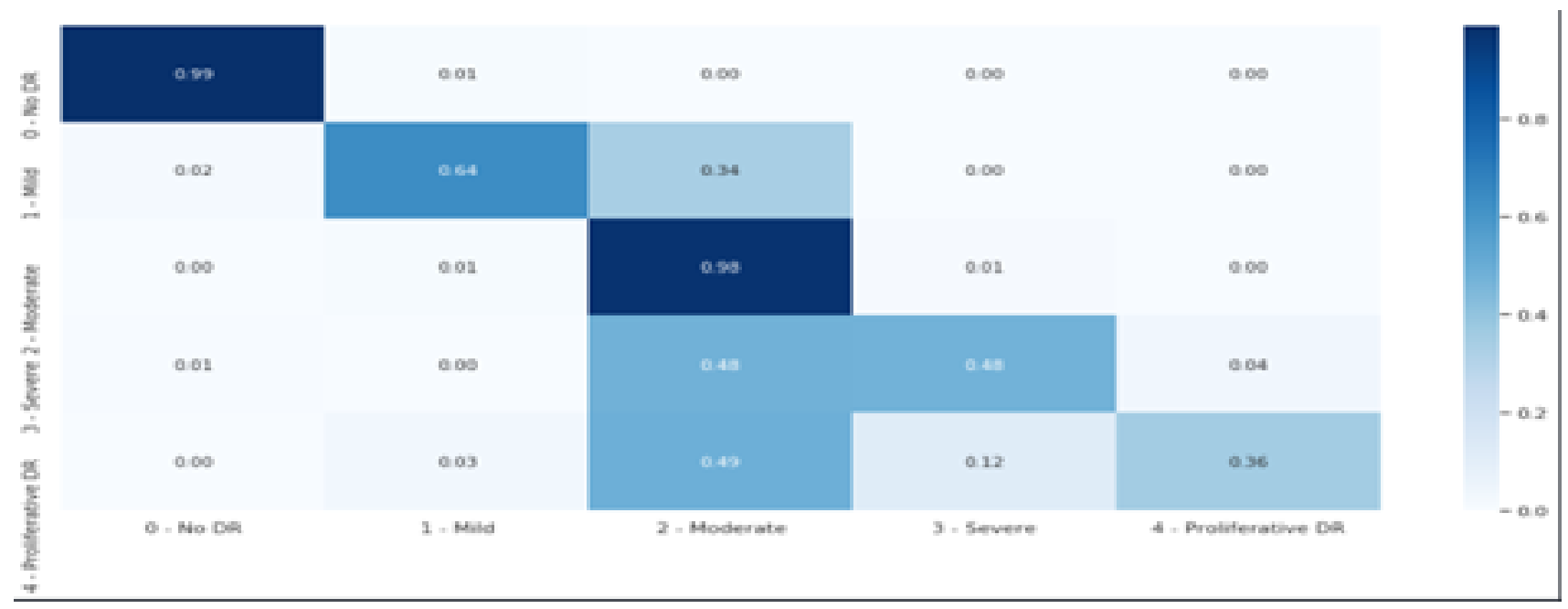

Figure 6: Shows the values of Confusion Matrix that summarizes the performance of the applied model in this research

\section{RESULTS}

In this research work, from publicly available dataset more than 1 million images have been trained in order to build the Convolutional Neural Network $(\mathrm{CNN})$. As pre-trained residual network such as ResNet50 have been used, this model works for millions of parameters. To validate the model, the system had been tested using thousands of images.

\subsection{Model Evaluation}

Model Loss Graph constitutes the loss and the accuracy for classification problems as well as the loss and accuracy for the validation dataset. In this context, Figure 5 represents the best quadratic kappa scores for epochs 13, 20, 14 respectively as around 22 numbers of epochs was taken while training and validating the dataset. From the model considering the time, values of training loss, validation loss and quadratic kappa had also been estimated.

\subsection{Confusion Matrix}

In this work a confusion matrix has been used for summarizing the performance of the CNN model ResNet 50 while detecting Diabetic Retinopathy (DR). Figure 6 illustrates it in more details that clearly indicates much better predictive model for DR classification problems.

\section{CONCLUSION}

This paper has worked towards a dynamic classification problem solving approach regarding detection of Diabetic Retinopathy (DR) using Convolutional Neural Network $(\mathrm{CNN})$. The proposed model can handle more than 25 million parameters. So it can be concluded that this model to the best of the authors' knowledge is significantly better at performance than pre-existing models considering time memory complexity and the accuracy specificity of the results.

\section{REFERENCES}

[1] Gurudath, Nikita, Celenk, Mehmet and Riley, H. 2015. Machine learning identification of diabetic retinopathy from fundus images. 2014 IEEE Signal Processing in Medicine and Biology Symposium, IEEE SPMB 2014 Proceedings. 10.1109/SPMB.2014.7002949.

[2] Joshi, S. and Karule, P.T., 2012. Retinal blood vessel segmentation. International Journal of Engineering and Innovative Technology (IJEIT), 1(3), pp.175-178.
[3] Dhanushkodi, S.S.R. and Vasuki, M., 2013. Diagnosis system for diabetic retinopathy to prevent vision loss. Applied Medical Informatics, 33(3), pp.1-11.

[4] Sevik, U., Kose, C., Berber, T. and Erdol, H., 2014. Identification of suitable fundus images using automated quality assessment methods. Journal of biomedical optics, 19(4), p.046006.

[5] Sujith Kumar, S.B. and Singh, V., 2012. Automatic detection of diabetic retinopathy in non-dilated RGB retinal fundus images. International Journal of Computer Applications, 47(19).

[6] Abràmoff, M.D., Folk, J.C., Han, D.P., Walker, J.D., Williams, D.F., Russell, S.R., Massin, P., Cochener, B., Gain, P., Tang, L. and Lamard, M., 2013. Automated analysis of retinal images for detection of referable diabetic retinopathy. JAMA ophthalmology, 131(3), pp.351-357.

[7] Priya, R. and Aruna, P., 2012. SVM and neural network based diagnosis of diabetic retinopathy. International Journal of Computer Applications, 41(1).

[8] Bragge, P., Gruen, R.L., Chau, M., Forbes, A. and Taylor, H.R., 2011. Screening for presence or absence of diabetic retinopathy: a meta-analysis. Archives of Ophthalmology, 129(4), pp.435-444.

[9] Casanova, R., Saldana, S., Chew, E.Y., Danis, R.P., Greven, C.M. and Ambrosius, W.T., 2014. Application of random forests methods to diabetic retinopathy classification analyses. PLoS One, 9(6), p.e98587.

[10] Garg, S., Jani, P.D., Kshirsagar, A.V., King, B. and Chaum, E., 2012. Telemedicine and retinal imaging for improving diabetic retinopathy evaluation. Archives of internal medicine, 172(21), pp.1677-1680.

[11] Lazar, I., Qureshi, R.J. and Hajdu, A., 2010, October. A novel approach for the automatic detection of micro aneurysms in retinal images. In 2010 6th International Conference on Emerging Technologies (ICET) (pp. 193197).IEEE.

[12] Sánchez, C.I., Hornero, R., López, M.I., Aboy, M., Poza, J. and Abásolo, D., 2008. A novel automatic image processing algorithm for detection of hard exudates based on retinal image analysis. Medical engineering \& 
physics, 30(3), pp.350-357.

[13] Sinthanayothin, C., Boyce, J.F., Williamson, T.H., Cook, H.L., Mensah, E., Lal, S. and Usher, D., 2002. Automated detection of diabetic retinopathy on digital fundus images. Diabetic medicine, 19(2), pp.105-112.

[14] Torok, Z., Peto, T., Csosz, E., Tukacs, E., Molnar, A., Maros-Szabo, Z., Berta, A., Tozser, J., Hajdu, A., Nagy, V. and Domokos, B., 2013. Tear fluid proteomics multimarkers for diabetic retinopathy screening. BMC ophthalmology, 13(1), p.40.

[15] Gulshan, V., Peng, L., Coram, M., Stumpe, M.C., Wu, D., Narayanaswamy, A., Venugopalan, S., Widner, K., Madams, T., Cuadros, J. and Kim, R., 2016. Development and validation of a deep learning algorithm for detection of diabetic retinopathy in retinal fundus photographs. Jama, 316(22), pp.2402-2410.

[16] Gargeya, R. and Leng, T., 2017. Automated identification of diabetic retinopathy using deep learning. Ophthalmology, 124(7), pp.962-969.

[17] Poplin, R., Varadarajan, A.V., Blumer, K., Liu, Y., McConnell, M.V., Corrado, G.S., Peng, L. and Webster, D.R., 2017. Predicting cardiovascular risk factors from retinal fundus photographs using deep learning. arXiv 2017. arXiv preprint arXiv:1708.09843.

[18] Abràmoff, M.D., Lou, Y., Erginay, A., Clarida, W., Amelon, R., Folk, J.C. and Niemeijer, M., 2016. Improved automated detection of diabetic retinopathy on a publicly available dataset through integration of deep learning. Investigative ophthalmology \& visual science, 57(13), pp.5200-5206.

[19] Ting, D.S.W., Cheung, C.Y.L., Lim, G., Tan, G.S.W., Quang, N.D., Gan, A., Hamzah, H., Garcia-Franco, R., San Yeo, I.Y., Lee, S.Y. and Wong, E.Y.M., 2017. Development and validation of a deep learning system for diabetic retinopathy and related eye diseases using retinal images from multiethnic populations with diabetes. Jama, 318(22), pp.2211-2223.

[20] Xu, K., Feng, D. and Mi, H., 2017. Deep convolutional neural network-based early automated detection of diabetic retinopathy using fundus image. Molecules, 22(12), p.2054.

[21] Pratt, H., Coenen, F., Broadbent, D.M., Harding, S.P. and Zheng, Y., 2016. Convolutional neural networks for diabetic retinopathy. Procedia Computer Science, 90, pp.200-205.

[22] Yang, Y., Li, T., Li, W., Wu, H., Fan, W. and Zhang, W., 2017, September. Lesion detection and grading of diabetic retinopathy via two-stages deep convolutional neural networks. In International Conference on Medical Image Computing and Computer-Assisted Intervention (pp. 533-540). Springer, Cham.

[23] Gondal, W.M., Köhler, J.M., Grzeszick, R., Fink, G.A. and Hirsch, M., 2017, September. Weakly-supervised localization of diabetic retinopathy lesions in retinal fundus images. In 2017 IEEE International Conference on Image Processing (ICIP) (pp. 2069-2073).IEEE.

[24] Quellec, G., Charrière, K., Boudi, Y., Cochener, B. and Lamard, M., 2017. Deep image mining for diabetic retinopathy screening. Medical image analysis, 39, pp.178-193.

[25] Choi, J.Y., Yoo, T.K., Seo, J.G., Kwak, J., Um, T.T. and Rim, T.H., 2017. Multi-categorical deep learning neural network to classify retinal images: A pilot study employing small database. PloS one, 12(11), p.e0187336.

[26] Kermany, D.S., Goldbaum, M., Cai, W., Valentim, C.C., Liang, H., Baxter, S.L., McKeown, A., Yang, G., Wu, X., Yan, F. and Dong, J., 2018. Identifying medical diagnoses and treatable diseases by image-based deep learning. Cell, 172(5), pp.1122-1131.

[27] Wang, Z., Yin, Y., Shi, J., Fang, W., Li, H. and Wang, X., 2017, September. Zoom-in-net: Deep mining lesions for diabetic retinopathy detection. In International Conference on Medical Image Computing and Computer-Assisted Intervention (pp. 267-275). Springer, Cham.

[28] García, G., Gallardo, J., Mauricio, A., López, J. and Del Carpio, C., 2017, September. Detection of diabetic retinopathy based on a convolutional neural network using retinal fundus images. In International Conference on Artificial Neural Networks (pp. 635-642).Springer, Cham.

[29] Costa, P. and Campilho, A., 2017. Convolutional bag of words for diabetic retinopathy detection from eye fundus images. IPSJ Transactions on Computer Vision and Applications, 9(1), p.10.

[30] Wang, J. and Olson, E., 2016, October. AprilTag 2: Efficient and robust fiducial detection. In 2016 IEEE/RSJ International Conference on Intelligent Robots and Systems (IROS) (pp. 4193-4198).IEEE.

[31] Feinman, R., Curtin, R.R., Shintre, S. and Gardner, A.B., 2017. Detecting adversarial samples from artifacts.arXiv preprint arXiv:1703.00410.

[32] Zhang, W., Zhao, Y., Breckon, T.P. and Chen, L., 2017. Noise robust image edge detection based upon the automatic anisotropic Gaussian kernels. Pattern Recognition, 63, pp.193-205.

[33] Zhang, J., Zhang, T., Dai, Y., Harandi, M. and Hartley, R., 2018. Deep unsupervised saliency detection: A multiple noisy labeling perspective. In Proceedings of the IEEE Conference on Computer Vision and Pattern Recognition (pp. 9029-9038).

[34] Laddha, A., Kocamaz, M.K., Navarro-Serment, L.E. and Hebert, M., 2016, June. Map-supervised road detection. In 2016 IEEE Intelligent Vehicles Symposium (IV) (pp. 118-123).IEEE.

[35] Kaur, S. and Singh, I., 2016. Comparison between edge detection techniques. International Journal of Computer Applications, 145(15), pp.15-18.

[36] Routray, S., Ray, A.K. and Mishra, C., 2017, February. Analysis of various image feature extraction methods against noisy image: SIFT, SURF and HOG. In 2017 Second International Conference on Electrical, Computer and Communication Technologies (ICECCT) (pp. 1-5) IEEE. 